\title{
Heart Rate Variability Analysis is More Sensitive at Identifying Neonatal Sepsis than Conventional Vital Signs
}

Fredrick J. Bohanona, Amy A. Mrazeka, Mohamed T. Shabana, Sarah Mims a, Geetha L.

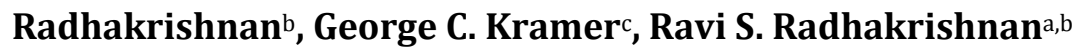

a Department of Surgery, b Department of Pediatrics, c Department of Anesthesiology, University of Texas Medical Branch, 301 University Blvd., Galveston, TX, 77555, USA

\section{Running Title: Heart Rate Variability is a Sensitive Indicator of Neonatal Sepsis}

\section{Subject Category: Critical Care}

Send correspondence to: Ravi S. Radhakrishnan

Department of Surgery

The University of Texas Medical Branch

301 University Boulevard

Galveston, Texas 77555-0353

Telephone: (409) 772-5666

FAX: (409) 772-4253

E-mail: rsradhak@utmb.edu

Presented at the Academic Surgical Congress Meeting, San Diego, California, February 4-6, 2014. 


\section{ABSTRACT}

Background: Sepsis remains the largest preventable source of neonatal mortality in the world. Heart rate variability (HRV) analysis and noninvasive cardiac output, have been shown to be useful adjuncts to sepsis detection in many patient groups.

Methods: With IRB approval, 4 septic and 6 nonseptic ELBW patients were enrolled. Data from septic and healthy patients were collected for 5 hours. ECG waveform and traditional vital signs were collected and the RR intervals were calculated, then heart rate variability analysis was performed in both the time- and frequency-domain.

Results: HRV measurements in time-domain, HR, and Sp02 were significantly different in septic patients vs. nonseptic controls

Conclusion: These results indicate that nonconventional vital signs such as HRV are more sensitive than traditionally used vital signs, such as CO and MAP, in the confirmation of sepsis in ELBW neonates. HRV may allow for earlier identification of septic physiology.

Key Words: heart rate variability, sepsis, neonatal, noninvasive vital signs 


\section{INTRODUCTION}

With the implementation of the Millennium Development Goals there has been a reduction in live born children deaths, before the age of 5, from 9.9 million in 2000 to 6.3 million in 2013 (1). Despite advances in neonatal critical care there were 2.76 million (44\%) deaths in the neonatal period (0-27 days) with sepsis accounting for 421,000 deaths (1). Prematurity and low birth weight exacerbate the risk for sepsis with $20-40 \%$ of premature infants having at least one episode of sepsis during their hospitalization, with higher incidences in the extremely low birth weight patients (ELBW, 401-1000 grams) $(2,3)$. In addition, sepsis leading to cardiovascular collapse results in significantly increased mortality rates, reaching $20 \%$, as well as increased hospital stay (3). In the patients who survive the episode of sepsis, the morbidity is significant, with $17 \%$ developing cerebral palsy and $15 \%$ with vision impairment $(4,5)$. Early recognition and appropriate goal-directed therapy remain the foundations for successful sepsis therapy.

Blood culture is the gold standard for diagnosis of sepsis; however it can take up to 48 hours for diagnosis and is associated with false positive and negative results $(6,7)$. The length of time needed for positive culture limits the ability to initiate and treat neonatal sepsis appropriately. Currently, blood pressure, heart rate and pulse oximetry are objective measures of shock, while capillary refill is a subjective tool. However, these metrics provide limited information as to the severity of shock, need for blood transfusion and as a means to assess resuscitation with fluids and drugs (8). A variety of "new vital signs" are emerging in medicine (9-11). We define "New Vital Sign" to be a physiologic variable that, until recently, has not been available for clinical care. Examples of these new vital signs are tissue oxygenation and heart rate variability (HRV) (12-17). Additional new vital signs are clinical variables that could only be intermittently or invasively measured with traditional technology, but now can be measured continuously and noninvasively, such as cardiac output $(18,19)$. HRV has been shown, prospectively, to have a high negative predictive value with a low positive predictive value $(\sim 15 \%)$ in identifying neonatal patients at high risk for sepsis and 
death. This positive predictive value improves with the incorporation of laboratory values $(20,21)$. Studies evaluating cardiac diseases (heart failure, hypertension, and myocardial infarction) have shown HRV analysis to be a widespread tool to study the interaction between the sympathetic and parasympathetic effects on heart rate. There are 2 main ways to evaluate in HRV: time and frequency domain analysis. Time domain indices are derived from statistical methods from the beat-to-beat interval (RR). These indices include standard deviation of RR interval (SDRR), percentage of absolute difference between consecutive RR intervals that are greater than 50ms (pNN50), two standard descriptors to evaluate short- and long-term variability (SD1 and SD2, respectively), and assessment of autonomic input via cardiac sympathetic and vagal index (CSI and CVI, respectively $(22,23)$. Frequency domains are a series of indices that evaluate the power spectral density of the contribution of the autonomic nervous system. These frequencies are divided into Very Low Frequency (VLF), Low Frequency (LF) and High Frequency (HF) power, with studies suggesting that HF is associated with parasympathetic activity and LF is associated with sympathetic activity (24). This is the first study to evaluate the time and frequency domain assessment of HRV along with noninvasive cardiac output monitoring in septic neonates.

\section{METHODS}

\section{Patients}

Data were obtained from a cohort of extremely low birth weight (ELBW, $<1000$ grams) neonates hospitalized at the Infant Special Care Unit (ISCU) at the University of Texas Medical Branch (UTMB), Galveston, Texas from August 2012 to May 2013. Inclusion criteria included ELBW and less than one month of age. Exclusion criteria included any major chromosomal abnormalities, cardiac defects, or weight more than 1000 gm. This study consisted of two cohorts of patients; healthy patients that met the inclusion criteria and a set of septic patients that had been diagnosed with sepsis within the preceding twelve hours prior to data collection. This study was approved by the Institutional Review Board of UTMB. Parents were informed and consent was obtained. 


\section{Data Collection}

The definition of sepsis was based on the sepsis continuum definition proposed by the International Pediatric Sepsis Consensus Conference in 2002 with the modification proposed by Wynn et al. to include preterm infants $(25,26)$. This sepsis continuum definition includes definitions for systemic inflammatory response syndrome (SIRS), infection, sepsis, severe sepsis, and septic shock. The organ system dysfunction definitions to differentiate sepsis from severe sepsis or septic shock will be taken from the same consensus conference.

Traditional vital signs were collected using Philip IntelliVue (model) monitor (Philips Healthcare, Andover, MA) and Cardiotronic Aesculon monitor (Osypka Medical Inc., La Jolla, CA.). Electrocardiogram (ECG) data was acquired by PowerLab and was analyzed via LabChart software (ADInstruments, Inc., Colorado Springs, CO.). Time- and frequency domain HRV analyses were performed using Nevrokard aHRV software (Nevrokard Kiauta, d.o.o, Slovenia).

All patients enrolled in this study had 5 hours of continuous vital signs and ECG data collection. Vital signs were recorded per standard of care. Healthy patients had the 1 st 5 hours after placement of electrodes and monitors collected, while the septic patients had the $1^{\text {st }} 5$ hours after confirmation of diagnosis.

\section{Statistical Analysis}

Five hours of continuous data was collected and the median value for each parameter studied was used for each patient. Parameters studied were HR, CO, mean-arterial pressure (MAP), pulse oximetry, index of contractility (ICON), SDRR, pNN50, SD1, SD2, CSI, CVI, VLF, LF, HF and LF/HF ratio. The median values were pooled into the 2 study groups and analyzed using an unpaired Student's T-test (GraphPad Prism 5.0, Graphpad Software Inc., La Jolla, CA.). Statistical significance was achieved if $\mathrm{P}<0.05$.

\section{RESULTS}

\section{Study Population}


Table 1 shows the demographic characteristics of the neonates studied. There were $(n=11)$ culture positive episodes in 4 patients. There were 6 healthy non-septic neonates as a control population. There was no statistical difference in age between healthy controls and septic patients $(27.33 \pm 0.67$ weeks vs $26.75 \pm 1.37$ weeks, $\mathrm{P}=0.68) .67 \%$ of healthy controls and $75 \%$ of septic patients were female $(\mathrm{P}=0.80)$. Birth weight was $643.00 \pm 101.1 \mathrm{~g}$ in healthy controls vs $697.8 \pm$ $113.8 \mathrm{~g}$ in septic patients $(\mathrm{P}=0.73)$. There was no statistical difference in length of stay (LOS) between healthy controls and septic patients $(110.00 \pm 17.65$ days vs $92.00 \pm 37.12$ days, $\mathrm{P}=0.64$, respectively). No patients died in the control group, while 2 (50\%) died in the septic group.

\section{Traditional Vital Signs Analysis}

Heart rate analysis revealed a significant increase in heart rate in septic neonates when compared to healthy controls $(168.0 \pm 5.5 \mathrm{bpm}$ vs $152.3 \pm 4.2 \mathrm{bpm}, \mathrm{P}<0.05$, Figure $1 \mathrm{a})$. There was no difference in CO $(0.188 \pm 0.006 \mathrm{~L} / \mathrm{min}$ vs $0.187 \pm 0.006 \mathrm{~L} / \mathrm{min}, \mathrm{P}=0.97$, Figure $1 \mathrm{~b})$, ICON $(76.25$ $\pm 8.751 / \mathrm{s}$ vs $125.5 \pm 20.891 / \mathrm{s}, \mathrm{P}=0.10$, Figure $1 \mathrm{c})$ or MAP (45.25 $\pm 5.39 \mathrm{mmHg}$ vs $58.00 \pm 10.04$ $\mathrm{mmHg}, \mathrm{P}=0.33$, Figure $1 \mathrm{~d}$ ) between the septic and healthy groups. SpO2 of septic neonates was significantly reduced to $93.00 \pm 0.82 \%$ compared to $97.00 \pm 0.68 \%$ in healthy controls $(\mathrm{P}=0.005$, Figure 1e).

\section{New Vital Signs Analysis}

Sepsis greatly influenced time domain indices of HRV analysis. SDRR was significantly reduced in the setting of sepsis compared to healthy neonates $(29 \pm 5.3 \mathrm{~ms}$ vs $63 \pm 10 \mathrm{P}=0.04$, Figure $2 \mathrm{a}$ ). Figure $2 \mathrm{~b}$ illustrates that sepsis decreased the percent of RR intervals greater than 50 ms (pNN50) compared to healthy controls $(0.57 \pm 0.15 \%$ vs $5.04 \pm 1.56 \%$, $\mathrm{P}<0.05)$. Short-term variability (SD1) was not changed in the presence of sepsis $(21.71 \pm 5.34$ vs $40.41 \pm 7.00, P=0.09$, Figure 2c). Sepsis reduced long-term variability (SD2) significantly, $34.17 \pm 5.30$, compared to control, $74.35 \pm 12.85(\mathrm{P}<0.05$, Figure $2 \mathrm{~d})$. The ratio of SD1/SD2, therefore, was significantly increased in septic patients compared to healthy patients $(0.74 \pm 0.04$ vs $0.48 \pm 0.05, P=0.007$, 
Figure 2 e). Sepsis greatly reduced CSI $(1.37 \pm 0.07)$ as compared to control $(2.18 \pm 0.22, P=0.02$, Figure 2f), but sepsis didn't influence CVI (Figure 2g).

VLF was unchanged in the septic patients compared to controls $\left(11.99 \pm 9.00 \mathrm{~ms}^{2}\right.$ vs $42.83 \pm$ $12.96 \mathrm{~ms}^{2}, \mathrm{P}=0.11$, Figure 3a). Figure $3 \mathrm{~b}$ demonstrates a downward, although not significant, reduction in LF in septic $\left(7.77 \pm 3.77 \mathrm{~ms}^{2}\right)$ vs healthy $\left(63.41 \pm 22.43 \mathrm{~ms}^{2}, \mathrm{P}=0.08\right)$. Similarly, HF was reduced but not significantly in sepsis $(13.75 \pm 5.77 \mathrm{~ms} 2)$ vs healthy $(104.5 \pm 46.18, \mathrm{P}=0.15$, Figure 3c). LF/HF ratio was unchanged in the setting of sepsis (Figure 3d).

\section{DISCUSSION}

Sepsis in ELBW neonates significantly increased HR and decreased SpO2, but had no significant effect on CO, ICON, or MAP. Further, septic neonates had altered HRV analysis characterized with significant changes in the time domain and while there was marked reduction in frequency domain, it was not statistically significant.

Sepsis is a major cause of mortality in ELBW neonates and has estimated cost, in the United States, of $\$ 700$ million, and the treatment and long-term outcomes haven't changed in decades (27, 28). The need for accurate and timelier diagnosis of sepsis is needed. In this present study we compare traditional vital signs and "new vital signs," which include non-invasive monitoring of CO and ICON plus high resolution HRV analysis between culture positive septic ELBW neonates and health ELBW neonates. Sepsis and cardiovascular function in ELBW neonates are understudied in the literature. Of the limited studies on septic ELBW infants, the cardiovascular function is mainly assessed by echocardiography (29). Noninvasive cardiac monitoring is an emerging technique that has been studied and validated in adults and infants $(19,30-34)$. To our knowledge, this is the first study examining noninvasive cardiac monitoring in septic ELBW neonates. Our novel findings suggest that in the first few hours of sepsis the neonate maintains an adequate perfusion pressure mainly through increased HR not through an increase in contractility (increased stroke volume). This adaption, although not great enough to set off alarms on the bedside monitor, is able to 
maintain an adequate MAP as compared to the control patients. Additionally, we compared high resolution HRV between septic and healthy ELBW neonates and found that the time domain was more sensitive than frequency domain analysis in identifying sepsis.

Time domain indices are the simplest to calculate and use similar methods for calculation, making them comparable between different researchers. Time domain indices consist of statistical measures of the inter-beat interval (IBI). SDRR is the standard deviation of the IBI and accounts for all factors that contribute to HRV, and is associated with lower frequency power (35). pNN50 is percentage of neighboring IBI that differ from each other by greater than $50 \mathrm{~ms}$ and is associated with HF power (36). In this study, septic neonates had significant decreased SDRR and pNN50, that in age-adjusted studies predict morbidity and mortality (35). Poincare analysis (SD1, SD2) is derived from non-linear dynamics and illustrates the RR interval fluctuations (36). SD1 is related to the short-term beat-to-beat HRV (associated with HF), while SD2 describes long-term HRV (associated with LF) and the ratio delineates the relationship between the two. ELBW septic neonates demonstrated a greater SD2 than SD1 as compared to healthy neonates, and this correlated with the SDRR, in that sepsis has a greater influence on LF. These findings are similar to an animal study examining HRV after injection of endotoxin (37). CSI (SD2/SD1) and CVI (log (SD1xSD2)) are additional values calculated from the Poincare plot (38). Septic neonates' HRV may be more dependent upon the changes in sympathetic output than parasympathetic output.

Frequency domain analysis is a much more powerful tool to examine the autonomic contribution to HRV. HF band represents parasympathetic input. The LF band is less understood and is thought to have input from both sympathetic and parasympathetic inputs, and VLF has been associated with all-cause mortality $(35,36)$. In this limited study, there was no detectable difference in the frequency domain between septic and healthy neonates, and therefore frequency domain analysis may be less sensitive than time domain analysis in sepsis. 
Sepsis in ELBW neonates is a poorly studied disease and demands more rigorous study. In this present study, we demonstrate that high resolution HRV analysis and noninvasive monitoring of "new vital signs" is a sensitive tool to detect the minimal physiological changes in these patients, although it should be stated that in this present study we are unable to determine if these parameters are useful for the early diagnosis of sepsis. This study only evaluated HRV after a clinical diagnosis of sepsis was made and demonstrated that there are significant physiologic changes in the first hours of sepsis. Further studies of the hours prior to the patient becoming septic are needed and these results may allow for an earlier diagnosis and treatment, which may lead to decreased morbidity and mortality.

\section{ACKNOWLEDGEMENTS}

This work was supported by grants T32-GM8256 (FJB) from the National Institutes of Health, Office of Naval Research N00014-12-C-0556, U.S. Army W23RYX0104N605000, and the U.S. Army Medical Research Materiel Command under Award No. W81XWH-14-2-0161. Opinions, interpretations, conclusions and recommendations are those of the author and are not necessarily endorsed by the U.S. Army. We would also like to thank Karen Martin for her generous help in preparing our data for publication.

\section{REFERENCES}

[1] Liu L, Oza S, Hogan D, et al. Global, regional, and national causes of child mortality in 2000-13, with projections to inform post-2015 priorities: an updated systematic analysis. Lancet. 2015;385:430-40.

[2] Manzoni P, Rizzollo S, Decembrino L, et al. Recent advances in prevention of sepsis in the premature neonates in NICU. Early Hum Dev. 2011;87 Suppl 1:S31-3.

[3] Stoll BJ, Hansen N, Fanaroff AA, et al. Late-onset sepsis in very low birth weight neonates: the experience of the NICHD Neonatal Research Network. Pediatrics. 2002;110:285-91. 
[4] Alshaikh B, Yusuf K, Sauve R. Neurodevelopmental outcomes of very low birth weight infants with neonatal sepsis: systematic review and meta-analysis. J Perinatol. 2013;33:55864.

[5] Stoll BJ, Hansen NI, Adams-Chapman I, et al. Neurodevelopmental and growth impairment among extremely low-birth-weight infants with neonatal infection. JAMA. 2004;292:235765.

[6] Dong Y, Speer CP. Late-onset neonatal sepsis: recent developments. Arch Dis Child Fetal Neonatal Ed. 2015;100:F257-63.

[7] Volante E, Moretti S, Pisani F, Bevilacqua G. Early diagnosis of bacterial infection in the neonate. J Matern Fetal Neonatal Med. 2004;16 Suppl 2:13-6.

[8] Funk D, Sebat F, Kumar A. A systems approach to the early recognition and rapid administration of best practice therapy in sepsis and septic shock. Curr Opin Crit Care. 2009;15:301-7.

[9] Cohen MJ. Use of models in identification and prediction of physiology in critically ill surgical patients. Br J Surg. 2012;99:487-93.

[10] Martin RS, Norris PR, Kilgo PD, et al. Validation of stroke work and ventricular arterial coupling as markers of cardiovascular performance during resuscitation. J Trauma. 2006;60:930-4; discussion 4-5.

[11] Griffin MP, Moorman JR. Toward the early diagnosis of neonatal sepsis and sepsis-like illness using novel heart rate analysis. Pediatrics. 2001;107:97-104.

[12] Beuchee A, Carrault G, Bansard JY, et al. Uncorrelated randomness of the heart rate is associated with sepsis in sick premature infants. Neonatology. 2009;96:109-14. 
[13] Chang KL, Monahan KJ, Griffin MP, et al. Comparison and clinical application of frequency domain methods in analysis of neonatal heart rate time series. Ann Biomed Eng. 2001;29:764-74.

[14] Lake DE, Fairchild KD, Moorman JR. Complex signals bioinformatics: evaluation of heart rate characteristics monitoring as a novel risk marker for neonatal sepsis. J Clin Monit Comput. 2014;28:329-39.

[15] Nardi O, Polito A, Aboab J, et al. StO(2) guided early resuscitation in subjects with severe sepsis or septic shock: a pilot randomised trial. J Clin Monit Comput. 2013;27:215-21.

[16] Wang CY, Chuang ML, Liang SJ, et al. Diffuse optical multipatch technique for tissue oxygenation monitoring: clinical study in intensive care unit. IEEE Trans Biomed Eng. 2012;59:87-94.

[17] Rodriguez A, Lisboa T, Martin-Loeches I, et al. Mortality and regional oxygen saturation index in septic shock patients: a pilot study. J Trauma. 2011;70:1145-52.

[18] Nowak RM, Nanayakkara P, DiSomma S, et al. Noninvasive hemodynamic monitoring in emergency patients with suspected heart failure, sepsis and stroke: the PREMIUM registry. West J Emerg Med. 2014;15:786-94.

[19] Saugel B, Cecconi M, Wagner JY, Reuter DA. Noninvasive continuous cardiac output monitoring in perioperative and intensive care medicine. Br J Anaesth. 2015;114:562-75.

[20] Griffin MP, Lake DE, Moorman JR. Heart rate characteristics and laboratory tests in neonatal sepsis. Pediatrics. 2005;115:937-41.

[21] Griffin MP, Lake DE, Bissonette EA, et al. Heart rate characteristics: novel physiomarkers to predict neonatal infection and death. Pediatrics. 2005;116:1070-4. 
[22] Allen JJ, Chambers AS, Towers DN. The many metrics of cardiac chronotropy: a pragmatic primer and a brief comparison of metrics. Biol Psychol. 2007;74:243-62.

[23] Karmakar CK, Khandoker AH, Voss A, Palaniswami M. Sensitivity of temporal heart rate variability in Poincare plot to changes in parasympathetic nervous system activity. Biomed Eng Online. 2011;10:17.

[24] Billman GE. The LF/HF ratio does not accurately measure cardiac sympatho-vagal balance. Front Physiol. 2013;4:26.

[25] Wynn JL, Wong HR. Pathophysiology and treatment of septic shock in neonates. Clin Perinatol. 2010;37:439-79.

[26] Goldstein B, Giroir B, Randolph A, International Consensus Conference on Pediatric S. International pediatric sepsis consensus conference: definitions for sepsis and organ dysfunction in pediatrics. Pediatr Crit Care Med. 2005;6:2-8.

[27] Wynn JL, Wong HR, Shanley TP, et al. Time for a neonatal-specific consensus definition for sepsis. Pediatr Crit Care Med. 2014;15:523-8.

[28] Dhas BB, Antony HA, Bhat V, et al. Global DNA methylation in neonatal sepsis. Indian J Pediatr. 2015;82:340-4.

[29] Brierley J, Carcillo JA, Choong K, et al. Clinical practice parameters for hemodynamic support of pediatric and neonatal septic shock: 2007 update from the American College of Critical Care Medicine. Crit Care Med. 2009;37:666-88.

[30] Rajput RS, Das S, Chauhan S, et al. Comparison of Cardiac Output Measurement by Noninvasive Method with Electrical Cardiometry and Invasive Method with Thermodilution Technique in Patients Undergoing Coronary Artery Bypass Grafting. . World Journal of Cardiovascular Surgery. 2014;4:123-30. 
[31] Grollmuss O, Gonzalez P. Non-invasive cardiac output measurement in low and very low birth weight infants: a method comparison. Front Pediatr. 2014;2:16.

[32] Mtaweh H, Trakas EV, Su E, et al. Advances in monitoring and management of shock. Pediatr Clin North Am. 2013;60:641-54.

[33] Antonelli M, Bonten M, Cecconi M, et al. Year in review in Intensive Care Medicine 2012: III. Noninvasive ventilation, monitoring and patient-ventilator interactions, acute respiratory distress syndrome, sedation, paediatrics and miscellanea. Intensive Care Med. 2013;39:54357.

[34] Marik PE. Noninvasive cardiac output monitors: a state-of the-art review. J Cardiothorac Vasc Anesth. 2013;27:121-34.

[35] Shaffer F, McCraty R, Zerr CL. A healthy heart is not a metronome: an integrative review of the heart's anatomy and heart rate variability. Front Psychol. 2014;5:1040.

[36] Rajendra Acharya U, Paul Joseph K, Kannathal N, et al. Heart rate variability: a review. Med Biol Eng Comput. 2006;44:1031-51.

[37] Gholami M, Mazaheri P, Mohamadi A, et al. Endotoxemia is associated with partial uncoupling of cardiac pacemaker from cholinergic neural control in rats. Shock. 2012;37:219-27.

[38] Stephan-Blanchard E, Chardon K, Leke A, et al. Heart rate variability in sleeping preterm neonates exposed to cool and warm thermal conditions. PLoS One. 2013;8:e68211.

\section{FIGURE LEGENDS}

Figure 1: Sepsis Effects on Traditional Vital Signs in ELBW Neonates: Five hours of continuous vital signs were collected in septic and healthy neonates. Median values for HR (A), CO (B), ICON (C), MAP (D), and pulse oximetry (E) are represented in the bar graphs. Error bars represent SEM. 
Sepsis significantly decreased HR and pulse oximetry. Heart rate (HR), cardiac output (CO), index of contractility (ICON) and mean arterial pressure (MAP).

Figure 2: Sepsis Significantly Effects Time-Domain Heart Rate Variability Analysis: Five hours of continuous heart rates were analyzed in septic and healthy neonates. Median values are shown, error bars represent SEM. Sepsis significantly decreased SDRR (A), pNN50 (B), SD2 (D), SD1/SD2 (E), and CSI (F). Sepsis didn't have a statistically significant effect on SD1 (C) and CVI (G).

Figure 3: Sepsis Effects Frequency-Domain Heart Rate Variability Analysis: Five hours of continuous heart rates were analyzed in septic and healthy neonates. Median values are shown, error bars represent SEM. Sepsis had a non-statistically significant effect on VLF (A), LF (B), HF (C), LF/HF ratio (D). Very low frequency (VLF), low frequency (LF), high frequency (HF) power. 
Table 1: Demographics: Data for age, weight at birth and length of stay are mean \pm SEM

\begin{tabular}{|c|c|c|c|}
\hline & Non-septic $(n=6)$ & Septic $(n=4)$ & P-value \\
\hline $\begin{array}{l}\text { Gestational Age at } \\
\text { Birth (weeks) }\end{array}$ & $27.33 \pm 0.67$ & $26.75 \pm 1.37$ & 0.68 \\
\hline Sex-Female (\%) & $4(67)$ & $3(75)$ & 0.80 \\
\hline Birth Weight (g) & $643.00 \pm 101.1$ & $697.8 \pm 113.8$ & 0.73 \\
\hline $\begin{array}{l}\text { Length of Stay } \\
\text { (Days) }\end{array}$ & $110.00 \pm 17.65$ & $92.00 \pm 37.12$ & 0.64 \\
\hline Mortality (\%) & $0(0)$ & $2(50)$ & \\
\hline Source of Sepsis & & $\begin{array}{l}\text { Necrotizing enterocolitis (NEC), } \\
\text { spontaneous intestinal perforation, } \\
\text { Group B Streptococcus septicemia }\end{array}$ & \\
\hline Bacteria isolated & & $\begin{array}{l}\text { Escherichia coli, coagulase negative } \\
\text { Staphylococcus spp., Staphylococcus } \\
\text { epidermidis, Group B Streptococcus spp., } \\
\text { Enterobacter cloacae }\end{array}$ & \\
\hline
\end{tabular}



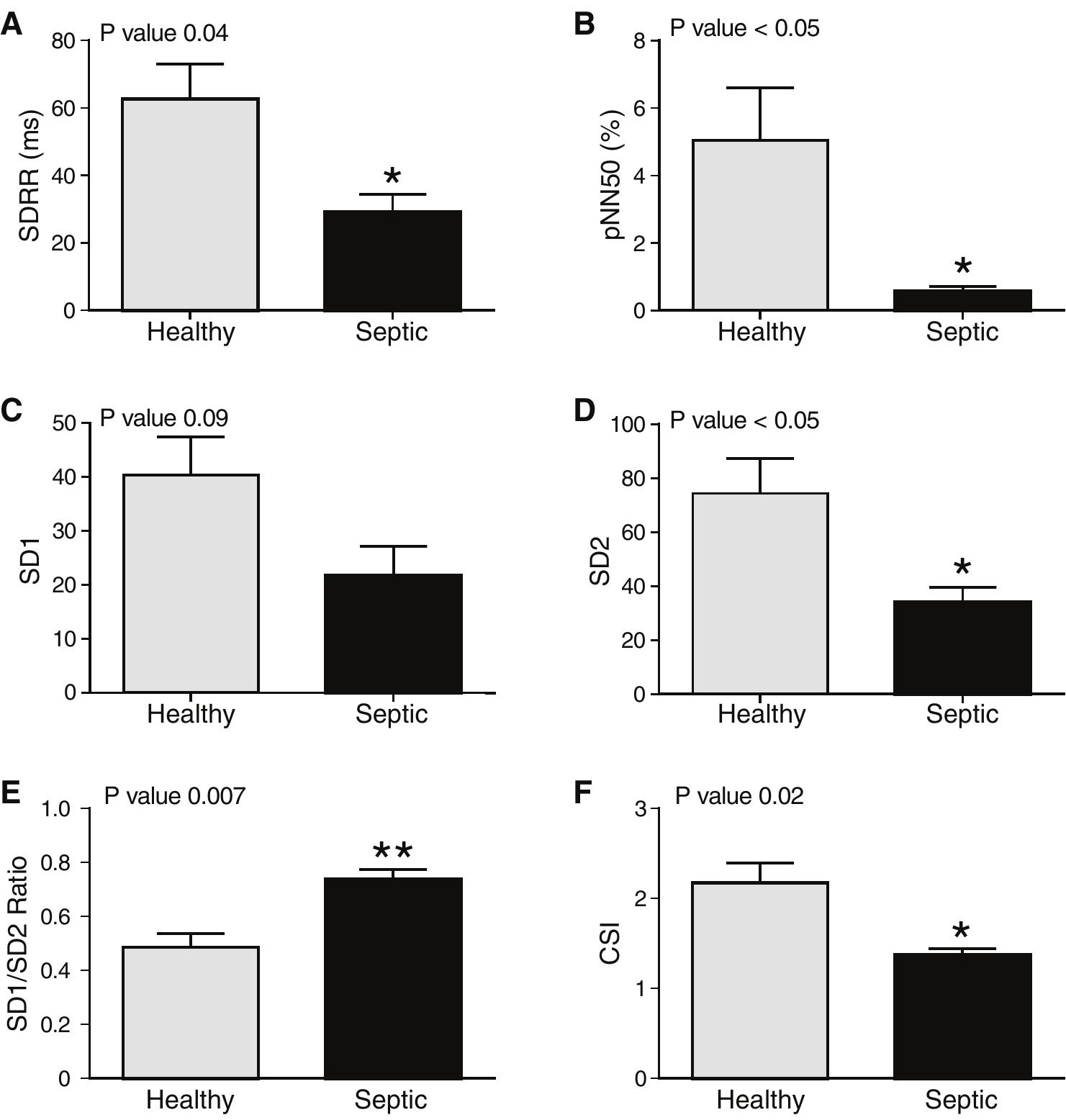

G $\quad 67 P$ value 0.06

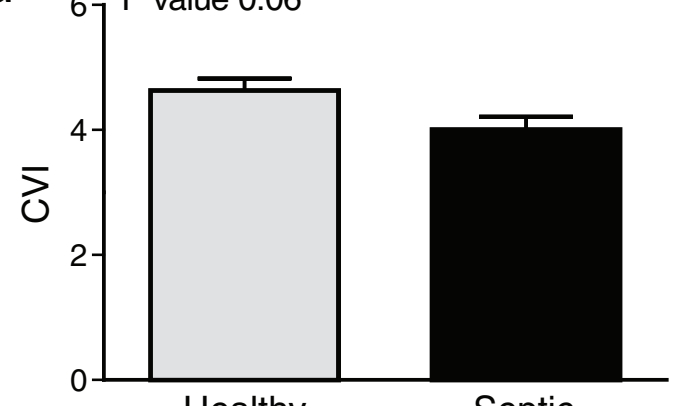

Septic 
Figure 3
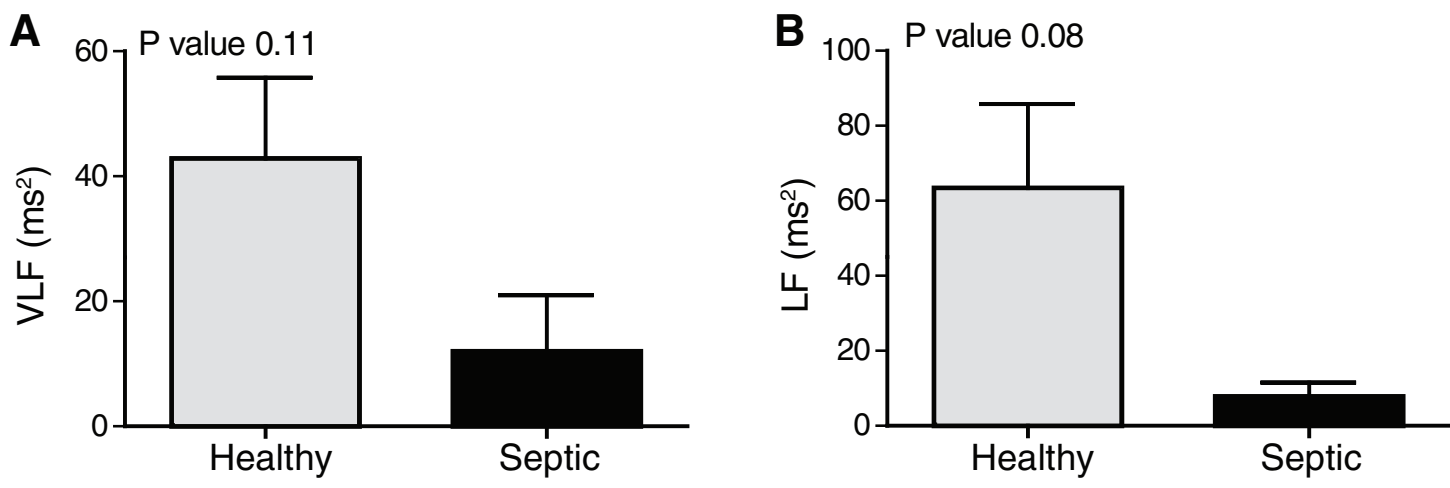

$\mathbf{C}_{200} \mathrm{P}$ value 0.15

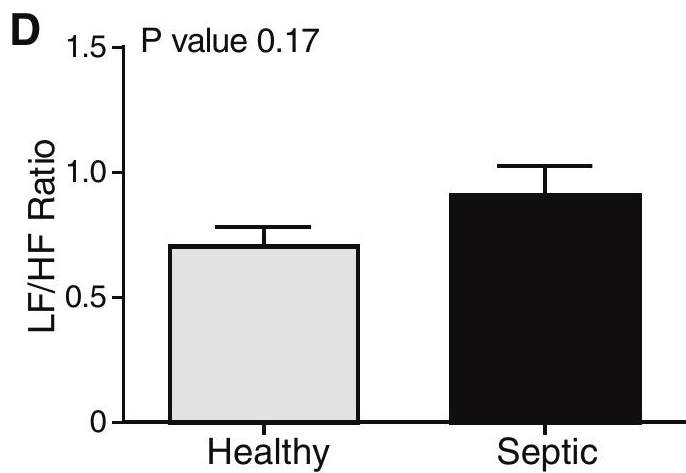

